CLAWAR 2020: 23rd International Conference on

Climbing and Walking Robots and the Support

Technologies for Mobile Machines,

Moscow, Russian Federation, 24-26 August 2020.

https://doi.org/10.13180/clawar.2020.24-26.08.37

\title{
MATHEMATICAL MODELING AND MOTION ANALYSIS OF CONVEYING OBJECT MOVING ON ROTATING SHAFTS
}

\author{
FUMIHIKO ASANO \\ School of Information Science, Japan Advanced Institute of Science and Technology, \\ 1-1 Asahidai, Nomi, Ishikawa 923-1292, Japan \\ E-mail: fasano@jaist.ac.jp
}

\section{Extended Abstract}

Toward novel technologies that contribute to factory automation, the author is now investigating the feasibility of the conveyor system illustrated in Fig. 1 (a); a thick disk-shaped object moves on two inclined rotating shafts using the effects of gravity and frictional forces at the contact points. In the future, we hope to realize more advanced object transportation by applying vibration to the entire stage.

In this paper, we consider a loupe-shaped conveying object illustrated in Fig. 1 (b); it has a shape where a circular upper part and a rod-shaped lower part are combined. Let $R[\mathrm{~m}]$ be the radius of the circular part, and $(x, y)$ be the central position of it. We assume that its center of mass $(\mathrm{COM})$ is located $l[\mathrm{~m}]$ below the two rotating shafts, and that the mass is $m$ $[\mathrm{kg}]$ and inertia moment around $\mathrm{COM}$ is $I\left[\mathrm{~kg} \cdot \mathrm{m}^{2}\right]$. It vibrates in the lateral plane according to the frictional force effect acting on the contact point with the two rotating shafts. The two rotating shafts are made of the same material, have the same radius, $r_{1}=r_{2}[\mathrm{~m}]$, and rotate at the same speed but in opposite directions. In addition, there is also a height difference between the two shafts; this is adjusted by $\phi_{1}$ and $\phi_{2}$.

Let $\boldsymbol{q}=\left[\begin{array}{lll}x & y & \theta\end{array}\right]^{\mathrm{T}}$ be the generalized coordinate vector. The equation of lateral motion and holonomic constraint condition become

$$
\boldsymbol{M} \ddot{\boldsymbol{q}}+\boldsymbol{h}=\boldsymbol{J}^{\mathrm{T}} \boldsymbol{\lambda}+\boldsymbol{J}_{\mu}^{\mathrm{T}} \boldsymbol{\lambda}, \quad \boldsymbol{J} \dot{\boldsymbol{q}}=\mathbf{0}_{2 \times 1},
$$

where $\boldsymbol{\lambda} \in \mathbb{R}^{2}$ is the constraint force vector acting on the central position of the circular part as shown in Fig. 1 (c). The term $\boldsymbol{J}_{\mu}^{\mathrm{T}} \boldsymbol{\lambda}$ is the frictional force vector, and can be

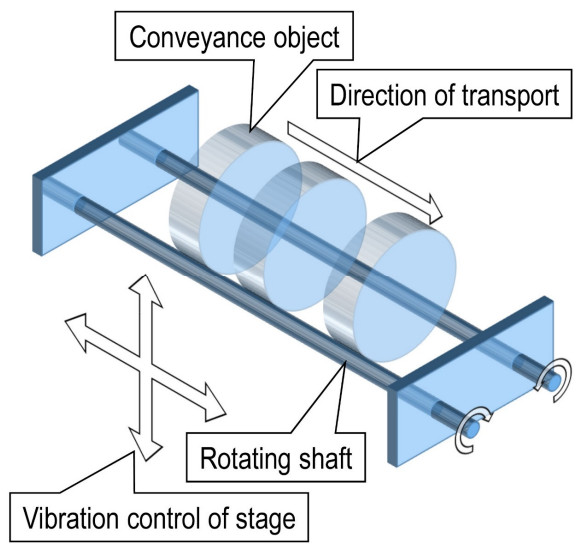

(a)

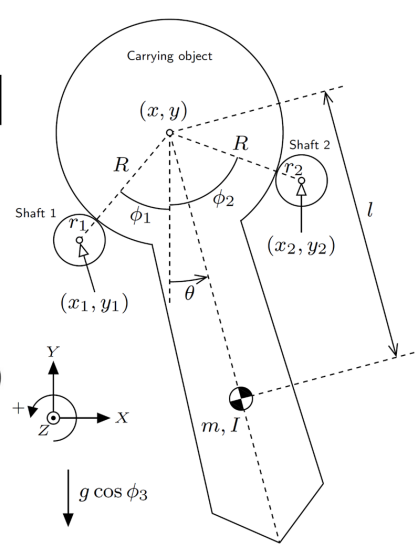

(b)

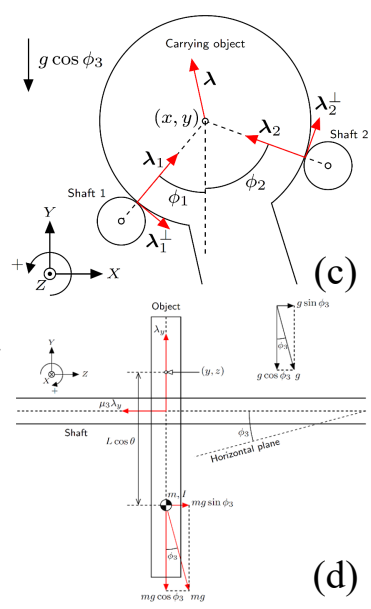

(d)

Fig. 1. Simulation results of object motion 


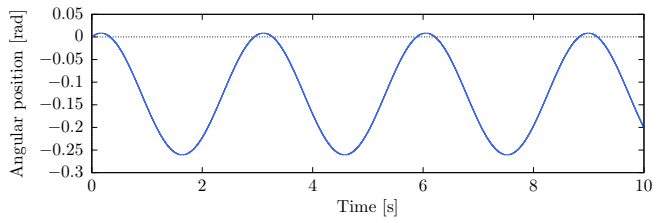

(a) $\theta$

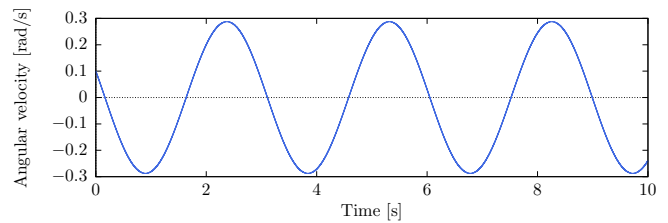

(b) $\dot{\theta}$

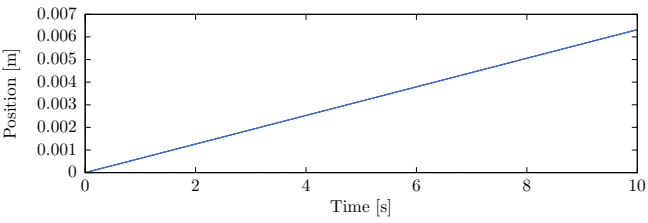

(c) $z$

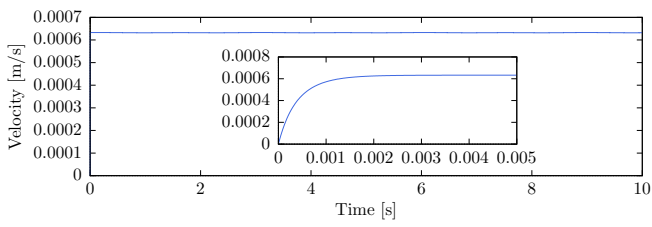

(d) $\dot{z}$

Fig. 2. Simulation results of object motion

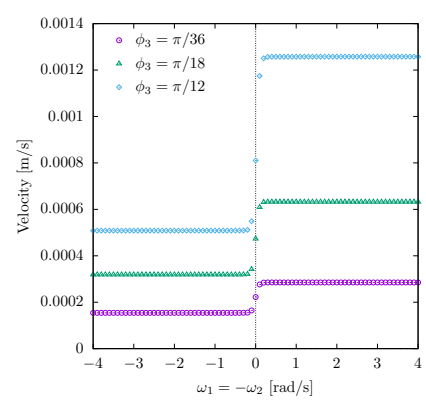

(a) Effect of $\omega_{1}$

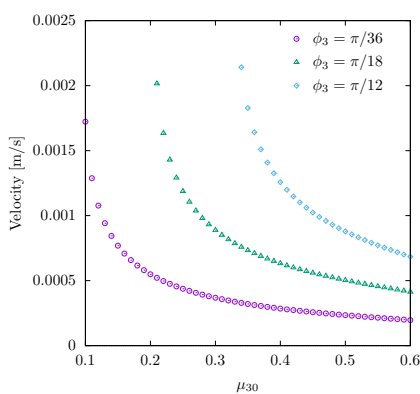

(b) Effect of $\mu_{30}$

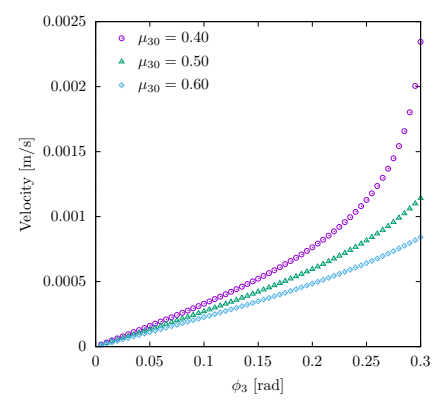

(c) Effect of $\phi_{3}$

Fig. 3. Conveyance speed versus system parameters

determined by resolving and rearranging the constraint force vector, $\boldsymbol{\lambda}$, as discussed in Ref. 1. The signed friction coefficient are specified as $\mu_{1}=-\mu_{10} \tanh \left(c\left(R \dot{\theta}+r_{1} \omega_{1}\right)\right)$ and $\mu_{2}=-\mu_{20} \tanh \left(c\left(R \dot{\theta}+r_{2} \omega_{2}\right)\right)$ where $\omega_{1}=-\omega_{2}[\mathrm{rad} / \mathrm{s}]$ are the rotating speed of the two shafts and are constant and $c$ is a positive constant for adjusting the shape of tanh. On the other hand, as illustrated in Fig. 1 (d), the equation of sagittal motion is simply modeled as

$$
m \ddot{z}=\mu_{3} \lambda_{y}+m g \sin \phi_{3},
$$

where $\mu_{3}=-\mu_{30} \tanh (c \dot{z})$. It is assumed that the motions in the lateral and sagittal planes do not interfere with each other.

Fig. 2 shows the simulation results of the object motion. We can see that the object moves forward at a constant sliding speed while vibrating laterally. Fig. 3 shows the analysis results of the effects of (a) $\omega_{1}$, (b) $\mu_{30}$, and (c) $\phi_{3}$. We can see that the conveyance speed is quickly saturated with the increase or decrease of $\omega_{1}$. We will report more detailed analysis results and basic experimental results in the presentation.

\section{Acknowledgments}

This research was partially supported by Grant-in-Aid for Scientific Research (C) No. 19K04255, provided by the Japan Society for the Promotion of Science (JSPS). The author would like to express his gratitude to GIKEN CO., LTD. for many helpful suggestions of the conveyor system.

\section{References}

1. F. Asano, Z. Luo, M. Yamakita and S. Hosoe, "Dynamic modeling and control for whole body manipulation," Pcoc. of the IEEE/RSJ Int. Conf. on Intelligent Robots and Systems, pp. 31623167, 2003. 